\title{
Pervasive refusal syndrome
}

\author{
Bryan Lask
}

Abstract Pervasive refusal syndrome is a severe, pervasive and life-threatening disorder. Most commonly seen in girls between the ages of 8 and 15, although also affecting boys and younger age groups, it is characterised by a profound and pervasive refusal to eat, drink, talk, walk and engage in any form of self-care. A determined resistance to treatment is a striking component of the condition. The causes are unclear, but likely to be complex, multiple and associated with a sense of hopelessness. Treatment needs to be comprehensive and is based on supporting the child in recovering at her own pace, while ensuring physical safety and well-being. The prognosis is good, provided treatment is appropriate, but recovery tends to take a year or more.

\section{Case example}

Anna, aged 14, the younger of two sisters, suffered a leg injury during training for an athletic event, at which she was the under-16 national champion. She had always seemed to enjoy running, and frequently trained with her parents, who were themselves dedicated and elite athletes. She had never expressed any lack of enthusiasm for running, although on one occasion had indicated that she disliked her coach, although unable to say why. She was doing very well at school, where she excelled in several subjects and was a very popular member of her peer group.

Conventional treatment for the leg injury had no effect, and eventually she was admitted to hospital for further investigation and treatment. By this time she was eating far less than usual and complaining of abdominal pain. Referral was made to a paediatrician who, after a few investigations, suggested a psychological assessment, noting that she had become increasingly withdrawn. Within a further 2 weeks she had become mute and immobile, was refusing to eat anything and angrily resisted all attempts to communicate with or help her.

\section{Background}

In 1991, my colleagues and I described four children, aged between 9 and 15, suffering from a potentially life-threatening condition, characterised by a profound and pervasive refusal to eat, drink, walk, talk or care for themselves in any way over a period of several months (Lask et al, 1991). All the children required extensive in-patient psychiatric care within a specialist residential child psychiatric unit, after deteriorating on paediatric units. Subsequently, numerous other reports appeared in the literature on a similar topic (e.g. Graham \& Foreman, 1995; Lask, 1996; Nunn \& Thompson, 1996; Thompson \& Nunn, 1997; Nunn et al, 1998; McGowan \& Green, 1998; Taylor et al, 2000). There has also been an account written by a parent describing the family's perspective (Anon, 2001). At the time of writing this article, I have been consulted on around 50 such cases around the world since our first description, in 1991.

\section{Definition}

Pervasive refusal syndrome is a condition involving varying degrees of refusal across several different domains, accompanied by dramatic social withdrawal and a determined resistance to treatment, leading to a seriously disabling and potentially lifethreatening condition. There is no evidence of organic disease. In 1997, Thompson \& Nunn suggested specific diagnostic criteria for this 'new' syndrome (Box 1$)$.

\section{Demography}

Pervasive refusal syndrome predominantly affects girls between the ages of about 8 and 16, although there have been two reports of its occurrence in boys (Taylor et al, 2000; Anon, 2001), one of whom was only 4 years old (Taylor et al, 2000). There has been

Bryan Lask is Professor of Child and Adolescent Psychiatry at St George's Hospital Medical School (Department of Psychiatry, Jenner Wing, Cranmer Terrace, London SW17 0RE, UK; e-mail: blask@sghms.ac.uk). He has worked with early-onset eating disorders for over 20 years and has researched widely into these conditions. He has published over 100 scientific papers and 7 books, and has lectured around the world. 
Box 1 Diagnostic criteria for pervasive refusal syndrome (Thompson \& Nunn, 1997)

- Clear food refusal and weight loss

- Social withdrawal and school refusal

- Partial or complete refusal in two or more of the following domains: mobilisation, speaking, attention to self-care

- Active and angry resistance to help or encouragement

- No organic condition to account for the severity or degree of symptoms

- No other psychiatric illness that could better account for the symptoms

no report of adults with the syndrome. There appears to be no social-class bias, and cases have been reported throughout Europe, North America and Australasia. The incidence is unknown, but the condition has become increasingly familiar to child and adolescent psychiatrists.

\section{Clinical features}

In most cases premorbid personality is either normal or characterised by high-achievement, high selfexpectation and difficulty coping with perceived failure. Commonly there is no significant past history, and the onset is relatively acute (Thompson \& Nunn, 1997). Occasionally the onset is more gradual, with a background of some emotional difficulties such as anxiety or somatisation. A past history of family illness is not uncommon, but it is unclear whether the prevalence exceeds that in the normal population.

The most common apparent precipitant factors are either a viral infection or some other intrusion such as an injury - in either instance there is not only a failure to respond to whatever might be the appropriate treatment, but also deterioration when treatment is implemented.

The presenting symptoms are those referred to in the definition above, but others have also been noted (Thompson \& Nunn, 1997). These include prominent somatic complaints, especially fatigue, lethargy, abdominal pain and nausea, for which no organic explanation can be found. In some cases depressed mood is detectable, but there are insufficient associated features to make a diagnosis of depression, and many of the presenting features are incompatible with such a diagnosis (e.g. the determined treatment refusal). Distress associated with parental separation on hospitalisation is common, although this is often later replaced with angry refusal to see the parents. When the children do speak, it is in a barely audible, highpitched voice, quite unlike their normal way of speaking. Their physical posture is most commonly the foetal position, with a tendency to be covered by bedclothes.

The presenting clinical picture is unlike that of any other condition and is comparable to that of a cornered, wounded and terrified animal. The children do not verbalise their distress, but enact it in their withdrawal, posture, refusal and resistance.

\section{Investigations}

These have almost all been normal, except for some abnormalities associated with low weight which reversed with weight restoration.

\section{Differential diagnosis}

Pervasive refusal syndrome appears to have many features in common with other conditions, but differs significantly from each of them and fails to meet the criteria for any of them. Furthermore, it has features that do not occur in other disorders. The following conditions (summarised in Box 2) need to be considered within the differential diagnosis.

\section{Depression}

Many of the features of pervasive refusal syndrome are compatible with a diagnosis of depressive disorder, but several aspects of it are not seen as part of that diagnosis, for example the determined treatment and other refusals. Common features of depression such as psychomotor slowing and sleep disturbance are uncommon in pervasive refusal syndrome. Furthermore, there is an enormous

\section{Box 2 Differential diagnosis}

Ten conditions should be considered:

- Depression

- Anxiety

- Eating disorders such as anorexia nervosa

- Selective mutism

- Catatonic disorders

- Stupor

- Somatoform disorder

- School refusal (school phobia)

- Chronic fatigue syndrome

- Factitious illness 
difference between children with the syndrome who do not have depressed mood and the far larger group of children who have depression but not the syndrome.

In childhood and adolescence there is considerable comorbidity associated with depression (Caron \& Rutter, 1991), and it is likely that some children with pervasive refusal syndrome do indeed also have comorbid depression. However, depressive disorder as a diagnosis is totally inadequate to account for the nature, depth and range of the symptoms.

\section{Anxiety}

Similar points might be made as in relation to depression. There do seem to be symptoms of anxiety, for example separation from parents and refusal or inability to attend school, but a diagnosis of anxiety alone could not account for the range and intensity of symptoms. The anxiety could be either a distinct and comorbid entity or a feature of pervasive refusal syndrome.

\section{Anorexia nervosa or other eating disorders}

Many, but not all, children with pervasive refusal syndrome present with typical features of anorexia nervosa. Others present with food refusal, but without the core features of anorexia nervosa (i.e. relentless pursuit of thinness and morbid preoccupation with weight and shape). The diagnosis of atypical eating disorder is often made. However, it soon becomes obvious that the refusal is neither particularly focused on, nor exclusive to food, nor are compensatory activities such as self-induced vomiting or excessive exercising evident.

\section{Selective mutism}

Although mutism is common in pervasive refusal syndrome, the other features exclude this diagnosis.

\section{Catatonic disorders}

The relative immobility, social withdrawal and mutism draw attention to the possibility of catatonia. However, the immobility of pervasive refusal syndrome is not associated with generalised psychomotor retardation, nor increased motor tone, and movement still occurs in sleep and when physical contact is attempted by those caring for the child. The social withdrawal is not associated with hypervigilance, hallucinations, delusions or thought disorder (Thompson \& Nunn, 1997). The mutism is either selective in nature or becomes so as recovery occurs.

\section{As Thompson \& Nunn have noted,}

'while there is perversity of response, there is no loss of purposive response. Determined, even combative opposition is quite different from the automaticity of negativism.'

\section{Stupor}

Although akinesis and negativism are common to both conditions, the wilful negativism of children with pervasive refusal syndrome, as well as the very gradual return of function over many months, make this diagnosis unlikely.

\section{Somatoform disorder}

Pervasive refusal syndrome also has much in common with somatisation disorder and conversion disorder. The multiple physical symptoms that affect several body systems but have no clear organic basis are reminiscent of somatisation disorder. However, there is no help-seeking behaviour: on the contrary, there is treatment resistance. Nor is it common in somatisation to be selectively mute, and to determinedly resist such activities as eating, showering and toileting. Similarly, although the inability to walk or move suggests conversion disorder, the wider range of symptoms exclude this diagnosis.

\section{School refusal (or school phobia)}

These children are all unable to go to school, but unlike those with school refusal, they are also unable or unwilling to participate in any other activity.

\section{Chronic fatigue syndrome}

Fatigue appears to be common in pervasive refusal syndrome, but it is by no means the most central feature. Children with chronic fatigue syndrome show very few of the other features of pervasive refusal syndrome, and particularly differ in that they express profound frustration with their ill-health and a strong wish to recover.

\section{Factitious illness}

Although children with pervasive refusal syndrome present with inexplicable symptoms, as do those with factitious illness, they differ from this latter group in that they retain virtually no spontaneous activity and vigorously avoid medical attention and care. This makes a diagnosis of factitious illness very unlikely. 


\section{Classification}

Pervasive refusal syndrome fails to fit neatly into any particular diagnostic category. This is in itself not an unusual phenomenon in child and adolescent psychiatry. Children are very inconsiderate in their refusal to fit into the great diagnostic schema. As Nichols et al (2000) have noted, 'Children into DSM don't go'. Indeed, in relation to eating disorders, both DSM-IV and ICD-10 show very poor interrater reliability. Pending further understanding, it is simply not possible to categorise this condition satisfactorily. At the moment, it tends to be considered alongside the eating disorders, but it could just as well be classified among any of the other conditions associated with refusal, for example school refusal or selective mutism. In time, it may prove possible to have a category of childhood conditions, the common feature of which is refusal (Nunn et al, 2000). For the meantime, pervasive refusal syndrome is best considered a descriptive label for a group of children who present with a constellation of clinical features which is distinct from other disorders (Thompson \& Nunn, 1997).

\section{Aetiology}

The causes of pervasive refusal syndrome are poorly understood. In the original sample (Lask et al, 1991), the possibility of sexual abuse seemed strong, but in later reports this has seemed less likely. Nunn \& Thompson (1996) have postulated that the syndrome might be explained using Seligman's model of learned helplessness, i.e. the learned expectation of having no capacity to control the environment, leading to a generalised passivity response. Some people feel helpless in the face of uncontrollability, whereas others do not. Those who do feel helpless may do so on the basis of the triad of personalisation, stability and globalisation: personalisation is the attribution of blame to oneself ('It's my fault'); stability is the view that it will always be this way ('Nothing can ever change'); and globalisation reflects the pervasiveness of the situation ('Every aspect of my life is ruined'). More recently, Seligman (1990) has stated that, to fulfil the criteria for learned helplessness, there must be (a) an experience of uncontrollability; (b) perception, explanation and expectation of uncontrollability; and (c) observable consequences in behaviour, particularly passivity.

Nunn \& Thompson (1996) have argued that pervasive refusal syndrome is a response to events that are perceived as uncontrollable, for example the loss of loved ones, any form of abuse, severe parental conflict, migration or frequent move of home and/or school. They develop the model by proposing that there is an interaction between parents and child as they watch one another struggling with events over which they perceive no control. Ultimately, the child experiences profound helplessness resulting from a loss of personal and vicarious hopefulness.

This model fits well with the aetiological paradigm of the three Ps - predisposing, precipitating and perpetuating factors. In pervasive refusal syndrome, the predisposing factor is the child's premorbid personality style (anxious, high-achieving, perfectionist), the precipitating factors are the events described above such as abuse or loss, and the perpetuating factors include the responses of the parents and clinicians to the child's somatic and refusal symptoms. Specifically, the children experience the therapeutic efforts as coercive, with subsequent reinforcement of their sense of having no control. This would explain why these children almost invariably deteriorate when active rehabilitation is attempted.

Other aetiological explanations are awaited.

\section{Treatment}

As with any other child psychiatric condition, treatment must be comprehensive, paying due attention at all times to organic, social and psychological factors. The condition is so severe that hospitalisation is almost always required. This should ideally be in a child/adolescent psychiatric unit, although a very few children have been successfully treated on a paediatric unit. There are as yet no reports of children with the syndrome being successfully treated as day patients or at home.

Some mainstays of treatment are listed in Box 3 and considered in greater in the following sections.

\section{Patience, time and sympathy}

On the basis of the learned helplessness paradigm it is self-evident that these children need very sensitive care. It takes a long time for such children

Box 3 Mainstays of treatment for pervasive refusal syndrome

- Patience, time and sympathy

- Basic nursing care offered by skilled staff

- The use of the ward milieu

- Physiotherapy

- Individual therapy

- Parental counselling/family therapy 
to recover, and pressurising them simply makes them worse. It is almost as if they need time out from normal life - a sabbatical. Indeed, most take about a year to recover from the time of implementation of appropriate treatment. It is a common experience among those who have cared for such children that therapeutic enthusiasm in the early stages is almost always counterproductive. Usually after a few months it becomes possible to implement a very gradual rehabilitation programme, but anything faster leads to regression, as many clinicians can testify.

It is essential to have skilled paediatric and psychiatric nurses, as well as a physiotherapist, dietician, and medical staff who are able to deal with both medical and psychiatric issues. There should also be staff with the skills both to communicate with silent children and to explore the possibility of abuse in all its ramifications.

\section{Nursing care and the ward milieu}

Nursing care is central to the programme. Nurses will be involved with all aspects of the children's care, including their physical, psychological, social, educational and spiritual needs. There should be a clear and structured management plan and a detailed daily timetable; each nurse should be familiar with this and able to apply it consistently with the other nurses and over time.

The nursing team is also integral to the use of the ward milieu as a safe, structured and consistent environment, in which the child can receive emotional and practical support. Nunn et al (1998) have provided a very detailed description of the moment-by-moment ward management of pervasive refusal syndrome.

Nasogastric feeds are almost always required to reverse dehydration, inadequate energy intake and electrolyte imbalance. When the tube and / or feeds are resisted, their implementation should always be done within the limits of parental consent or an appropriate legal framework (as, of course, should all aspects of the treatment). Neiderman et al (2001) have offered guidelines on nasogastric tube-feeding of children and adolescents with eating disorders.

Skin care includes frequent turning and the application of moisturising creams to prevent bedsores and infections.

\section{Physiotherapy and individual therapy}

Physiotherapy in the early stages is designed to release and prevent stiffness, to prevent contractures and to maintain as full a range of movement as possible. It includes passive stretches and ranging, and where possible the use of tilt-tables and hydrotherapy. Gradually, the child is encouraged to sit, later stand and eventually to resume walking.

Individual therapy plays a limited part in the early stages, but it should none the less be considered, as a prelude to the important role it plays later. The therapist needs to be skilled at communicating with mute children and comfortable with silence. The use of 'musing' - thinking aloud - seems to be helpful. The therapist gently and sympathetically considers what might be so troubling for the child and, in so doing, inwardly notes and attempts to make sense of the non-verbal responses. Use of toys, 'cuddlies' and art materials are all useful ways of communicating. The children usually find ways of conveying which, if any, of these techniques is acceptable and has utility. Later, but before speech returns, they may be willing to write letters or make use of contemporary technology such as e-mailing or text-messaging. The therapy best focuses on whatever material the child offers, rather than pursuing a specific agenda or therapeutic technique. Commonly the child is unable to consider what might have caused the condition, and is more likely to be concerned about the problems associated with returning to normal life.

\section{Parental counselling/family therapy}

The aims of parental involvement are to contain parental anxiety and distress, advise about management, promote consistency in management between the parents themselves and consistency between the parents and the clinical team. Family therapy allows inclusion of siblings, who themselves are likely to feel much distress and uncertainty about how to respond, as well as exploration and alleviation of potentially unhelpful family relationship and communication patterns.

\section{Medication}

Medication appears to have only a limited role in management. The use of antidepressants for comorbid depression may have value, but they do not seem to be universally helpful in all cases of pervasive refusal syndrome. Hopefully, time will tell whether it is indeed those cases where there is comorbid depression that seem to benefit from antidepressants.

Tranquillisers and sedatives should be used very sparingly. They are best reserved as an occasional and short-term measure for situations in which the child is inordinately distressed by such necessary procedures as nasogastric tube-feeding or passive physiotherapy. 


\section{The recovery process}

As the child starts to improve, the focus of treatment can change from containment and ensuring safety and physical health to very gradual rehabilitation, as is commonly used for chronic fatigue syndrome in both adults (e.g. Wessely et al, 1989) and children and adolescents (e.g. Richards, 2000). Although considerable attention must still be paid to the pace at which the child can improve, in rehabilitation there is far more focus on achieving goals. However, it cannot be overemphasised how important it is to gauge the child's pace.

Recovery is painfully slow and most children with pervasive refusal syndrome take about a year from the time of implementation of appropriate treatment. Generally, the symptoms that appear first are the last to disappear, so if food refusal was the first symptom, normal eating will not occur until all the other features have resolved. Similarly, the symptoms that are last to appear are usually the first to resolve. The most common of these is the mutism.

During recovery, children with the syndrome tend to pass through stages similar to those described in anorexia nervosa (Lask \& Bryant-Waugh, 1995). In stage one, the child presents all the features of pervasive refusal syndrome, but as she begins to recover she gradually enters stage two. In this stage there is marked assertiveness and the expression of considerable negative feeling, especially anger, towards those close to her. If this is allowed and supported, rather than discouraged and disapproved of, it gradually resolves and there is the emergence of normal age-appropriate feelings and behaviour (stage three). It is unclear whether stage 2 is a necessary stage of the recovery process, as has been claimed by Lask \& Bryant-Waugh for anorexia nervosa.

\section{Outcome}

Most children and adolescents with pervasive refusal syndrome appear to make a complete, or almost complete, recovery, with little residual pathology. The time taken to recover is remarkably slow, and the clinician's tendency is to try to speed it up. This almost invariably leads to regression and relapse.

\section{Discussion}

'Pervasive refusal syndrome' (Box 4) is a disorder identified only recently. With correct treatment, the prognosis for children with the syndrome is good. However, there are many unanswered questions: does the syndrome actually exist; are there less severe variants; what are the causes; what constitutes best practice?

Some of these questions have been addressed by Thompson \& Nunn (1997), who conclude that

\footnotetext{
'the term pervasive refusal syndrome remains a descriptive label for a group of children who present with a constellation of clinical features which is distinct from other related disorders. It is less clear that it has a unique aetiology, specific treatment and outcome, distinct from other disorders. Despite this uncertainty it is our opinion that the term PRS is clinically useful and certainly warrants further study.'
}

McGowan \& Green (1998) have suggested that the syndrome can present in milder variants, despite profoundly traumatic aetiology, perhaps because there was no family dysfunction or social deprivation. They also have drawn attention to the report by Brown \& Perkins (1989) of three girls aged 5-6 with a similar presentation of life-threatening severity. In these cases, however, uninhibited sexual play led to a rapid disclosure of sexual abuse. My colleagues and I have suggested that in older children the developmental decline in spontaneous play might make communication through this

\section{Box 4 Pervasive refusal syndrome: key points}

- Pervasive refusal syndrome is characterised by varying degrees of refusal across several different domains, accompanied by dramatic social withdrawal and a determined resistance to treatment

- This seriously disabling and potentially lifethreatening condition affects predominantly girls between the ages of about 8 and 15 years

- The causes are not known, but the children affected are likely to have a conscientious and perfectionist personality exposed to particular stresses over which they feel that they have absolutely no control, which leads to a state of learned helplessness

- The condition appears to be triggered by an incidental injury or infection within the context of a particularly difficult life situation

- Treatment is based on supporting the child in recovering at his or her own pace, while at the same time ensuring physical safety and well-being

- As recovery progresses, therapy can focus more on underlying concerns and the development of coping skills

- With correct treatment, the prognosis is good 
medium less likely, and that greater cognitive maturity might mean that the older child would weigh up more carefully the consequences of disclosure, be it of sexual abuse or of other intolerable stresses (Lask et al, 1991).

The causes remain unclear and require further consideration. It is possible that some of these children have indeed been sexually abused and even 'silenced', but this appears not to be the case in the majority. Nunn \& Thompson's hypothesis of learned helplessness seems to fit well with the majority of cases described. It has the additional merit of adequately explaining the development of the condition, without demanding specific precipitating factors. This would then account for the wide range of precipitants that have been reported.

Controversy exists regarding best treatment. This may be resolved by acknowledging that there are different stages to the condition. In the early stages, attempts to rehabilitate almost invariably lead to deterioration, whereas once recovery commences it is possible to introduce a graded rehabilitation programme without adverse effect.

There is an onus on all clinicians dealing with pervasive refusal syndrome to retain an open mind about its nature, aetiology and best treatment.

\section{References}

Anon (2001) Pervasive refusal syndrome: a parent's perspective. Clinical Child Psychology and Psychiatry, 6, 455-469.

Brown, R. \& Perkins, M. (1989) Child sexual abuse presenting as organic disease. BMJ, 299, 614-615.

Caron, C. \& Rutter, M. (1991) Co-morbidity in child psychopathology: concepts, issues and research strategies. Journal of Child Psychology and Psychiatry, 32, 1063-1080.

Graham, P. \& Foreman, D. (1995) An ethical dilemma in child and adolescent psychiatry. Psychiatric Bulletin, 19, 84-86.

Lask, B. (1996) Pervasive refusal syndrome. ACPP Occasional Papers, 12, 33-35.

Lask, B. \& Bryant-Waugh, R. (1995) Annotation - eating disorders in children. Journal of Child Psychology and Psychiatry, 36, 191-202.

Lask, B., Britten, C., Kroll, L., et al (1991) Children with pervasive refusal. Archives of Diseases in Childhood, 66, 866-869.

McGowan, R. \& Green, J. (1998) Pervasive refusal syndrome: a less severe variant with defined aetiology. Clinical Child Psychology and Psychiatry, 3, 583-590.

Neiderman, M., Richardson, J., Farley, A., et al (2001) Nasogastric feeding in early onset eating disorders. International Journal of Eating Disorders, 29, 441-448.

Nichols, D., Chater, R. \& Lask, B. (2000) Children into DSM don't go: a comparison of classification systems of eating disorders for children. International Journal of Eating Disorders, 28, 317-324.

Nunn, K. \& Thompson, S. (1996) The pervasive refusal syndrome: learned helplessness and hopelessness. Clinical Child Psychology and Psychiatry, 1, 121-132.

Nunn, K., Thompson, S., Moore, S., et al (1998) Managing pervasive refusal syndrome: strategies of hope. Clinical Child Psychology and Psychiatry, 3, 229-249.
Nunn, K., Nichols, D. \& Lask, B. (2000) A new taxonomy for child psychiatry. Clinical Child Psychology and Psychiatry, 5, 313-328.

Richards, J. (2000) Chronic fatigue syndrome in children and adolescents: a review article. Clinical Child Psychology and Psychiatry, 5, 31-51.

Seligman, M. (1990) Learned Optimism. New York: Knopf.

Taylor, S., Dossetor, D., Kilham, H., et al (2000) The youngest case of pervasive refusal syndrome? Clinical Child Psychology and Psychiatry, 5, 23-30.

Thompson, S. \& Nunn, K. (1997) The pervasive refusal syndrome: The Royal Alexandra Hospital for Children experience. Clinical Child Psychology and Psychiatry, 2, $145-165$

Wessely, S., David, A., Butler, S., et al (1989) Management of chronic (post-viral) fatigue syndrome. Journal of the Royal College of Practitioners, 39, 26-29.

\section{Multiple choice questions}

1 Pervasive refusal syndrome:

a affects the genders equally

b occurs in all socio-economic groups

c has not been reported in adults

$\mathrm{d}$ is particularly common in children from ethnic minorities.

2 Aetiology:

a pervasive refusal syndrome has been reported to be associated with trauma

b genetic factors have been identified

c birth injury is a recognised contributory factor

d learned helplessness is an important component.

3 Clinical presentation:

a sleep disturbance is common

b mutism rarely occurs

c food refusal always occurs

d treatment is resisted.

4 The following should be considered within the differential diagnosis:

a epilepsy

b affective disorder

c Munchausen's syndrome by proxy

d conversion disorder.

5 Treatment should include:

a early and active mobilisation

b interpretive psychotherapy

c parental counselling

d working at the child's pace.

\section{MCQ answers}

$\begin{array}{llllllllll}1 & & 2 & & 3 & & 4 & & 5 & \\ \text { a } & \text { F } & \text { a } & \text { T } & \text { a } & \text { F } & \text { a } & \text { F } & \text { a } & \text { F } \\ \text { b } & \text { T } & \text { b } & \text { F } & \text { b } & \text { F } & \text { b } & \text { T } & \text { b } & \text { F } \\ \text { c } & \text { T } & \text { c } & \text { F } & \text { c } & \text { T } & \text { c } & \text { F } & \text { c } & \text { T } \\ \text { d } & \text { F } & \text { d } & \text { T } & \text { d } & \text { T } & \text { d } & \text { T } & \text { d } & \text { T }\end{array}$

